\title{
Dietary microparticles and their impact on tolerance and immune responsiveness of the gastrointestinal tract
}

\author{
Jonathan J Powell*, Vinay Thoree and Laetitia C Pele \\ MRC Human Nutrition Research, Elsie Widdowson Laboratory, Fulbourn Road Cambridge, CB1 9NL, United Kingdom
}

Dietary microparticles are non-biological bacterial-sized particles of the gastrointestinal lumen that occur due to endogenous formation (calcium phosphate) or following oral exposure (exogenous microparticle). In the UK, about $40 \mathrm{mg}\left(10^{12}\right)$ of exogenous microparticles are ingested per person per day, through exposure to food additives, pharmaceutical/supplement excipients or toothpaste constituents. Once ingested, exogenous microparticles are unlikely to pass through the gastrointestinal tract without adsorbing to their surfaces some ions and molecules of the intestinal lumen. Both entropy and ionic attraction drive such interactions. Calcium ions are especially well adsorbed by dietary microparticles which then provide a positively charged surface for the attraction (adsorption) of other organic molecules such as lipopolysaccharides, peptidoglycans or protein antigen from the diet or commensal flora. The major (but not only) sites of microparticle entry into intestinal tissue are the M-cell rich lymphoid aggregates (termed Peyer's patches in the small bowel). Indeed, it is well established that this is an efficient transport route for non-biological microparticles although it is unclear why. We hypothesise that this pathway exists for "endogenous microparticles" of calcium phosphate, with immunological and physiological benefit, and that "exogenous dietary microparticles", such as titanium dioxide and the silicates, hijack this route. This overview focuses on what is known of these microparticles and outlines their potential role in immune tolerance of the gut (endogenous microparticles) or immune activation (exogenous microparticles) and inflammation of the gut.

Dietary microparticles: Immune responsiveness: Crohn's disease: Calcium phosphate and tolerance

Dietary microparticles are defined as non-biological particles of the gastrointestinal lumen that are of appropriate size and shape (typically $0 \cdot 2-1 \cdot 0 \mu \mathrm{m}$ diameter), for uptake by mucosal phagocytes, in much the same way as biological particles (i.e. bacteria) can be. It should be more widely appreciated that (i) two distinct types of microparticle exist, namely endogenous and exogenous ${ }^{1}$ (ii) gastrointestinal exposure is substantial (e.g. $10^{12}$ exogenous particles per person per day in the UK) ${ }^{2}$ (iii) specific mechanisms exist in the gut for the absorption of non-biological particles so tissue uptake is also large ${ }^{3}$ and (iv) such particles are most likely to adsorb or trap soluble molecules of the gut lumen so that cells are exposed to "conjugates" of non-biological particle plus associated biomolecules ${ }^{4-7}$. Over the past ten years our group has aimed to characterise these particles and to define their origins, route of gastrointestinal uptake, cellular fate and immuno-modulatory properties. We have also sought to test the hypothesis that microparticles are involved in the aetiopathogenesis of Crohn's disease. Here, we briefly overview exposure to and fate of dietary microparticles and then concentrate on what is known of their immune modulating properties.

\section{Exogenous or Endogenous?}

During early work, aimed at characterising the chemical behaviour of ingested metals in the gut lumen, Powell and colleagues noted that classically insoluble metal ions at intestinal pH (e.g. iron, aluminium, etc) were in fact rendered soluble by factors of the intestinal juices (ligands and mucin) whereas calcium, uniquely, was found in great abundance co-precipitated with phosphate ${ }^{8}$. These calcium phosphate precipitates had been well reported before ${ }^{9,10}$, but, what had not been well appreciated, is that a fraction of the precipitate is reasonably homogenous sub-micron sized microparticlesmaking these candidates for gut tissue uptake ${ }^{3,11}$. In fact the same group had already reported that when similarly sized particles are ingested, a proportion is taken up by the intestinal mucosa ${ }^{3}$. So, why might the gut "manufacture" microparticles of calcium phosphate in the lumen (i.e. endogenous particles) that could then be scavenged by mucosal phagocytes? And what would the impact be of exposing this same system to new, man-made microparticles (i.e. exogenous particles)?

Even on calcium and phosphorus-poor diets, active secretion of $\mathrm{Ca}^{2+}$ and $\mathrm{PO}_{4}^{3-}$ ions, from the intestinal mucosa and into the lumen, still occurs and co-precipitation ensues ${ }^{9,10,12}$. In spite of the observation that calcium phosphate microparticles (generally less than $1 \mu \mathrm{m}$ ) form in the gastrointestinal lumen, their translocation into the intestinal mucosa is yet to be shown. This is mainly due to technical challenges in preserving and observing freshly precipitated and thus labile, mineral structures in situ. Nonetheless, their presence can be invoked from the ubiquitous nature of calcium phosphate microparticles in the lumen and the exceptional 
capacity of $\mathrm{M}$ cells, which chiefly overlay intestinal lymphoid aggregates (Peyer's patches in the small bowel), in the uptake of non-biological particles ${ }^{11,13,14}$.

\section{Endogenous microparticles}

Our interest lies in the biological role of luminally precipitated calcium phosphate in the gut and, specifically, in the potential immunological benefit(s) of the microparticulate fraction. Calcium phosphate, due to its surface properties, is able to adsorb organic molecules and/or luminal components ${ }^{5-7}$ and therefore may play a role in luminal detoxification (through adsorption of faecal toxins). On quantitative grounds, however, a detoxification role seems unlikely for the microparticulate fraction. Instead, we recently proposed that calcium and phosphate ions may co-precipitate with luminal antigen forming a microparticle sized hydroxy-apatite-antigen complex which is then scavenged by M cells of Peyer's patches and passed to underlying phagocytic lymphoid cells for processing ${ }^{15}$. The presumption that the particles are hydroxy-apatite, rather than any other form of calcium phosphate, is based mainly upon in vitro models and needs to be carefully tested in vivo. If this mechanism is proven then we suggest the term "constitutive antigen transfection". This could represent a mechanism of immuno-surveillance by continual sampling of gut luminal contents and may lead to promotion of immunological tolerance.

How may calcium phosphate microparticles promote an immuno-tolerant response? It is now well recognised that dietary calcium, possibly through binding to luminal free fatty acids and bile acids, may confer protection against colonic carcinogenesis ${ }^{5-7,16}$. However, it has also recently been shown that dietary calcium modulates enteric infection and inflammation ${ }^{17-19}$, which is not easily explained through the binding of bile acids or fatty acids. Other studies have since provided convincing explanations. High levels of dietary calcium have been shown to increase apoptosis of the murine mid and distal colonic epithelial cells ${ }^{20,21}$ and to protect against intestinal weight loss and peripheral TNF- $\alpha$ production in an induced-colitis model $^{22}$. In agreement with these findings, we also demonstrated in a recent study that low dietary calcium levels modulate mucosal caspase expression and increase disease activity in mice with dextran sulfate sodium-induced colitis (unpublished results). Thus (by analogy to dietary calcium effects on intestinal epithelial cells), the first possible effect of calcium phosphate microparticles on intestinal mononuclear phagocytes is the induction of apoptosis. Indeed previous data indicate that luminal calcium levels of the gut are proportional to dietary levels ${ }^{1}$ and that the uptake of calcium phosphate microparticles by intestinal mononuclear cells can directly trigger cell death ${ }^{23}$. Thereafter tolerance could be established towards exogenous antigen contained within the apoptotic cells, as this would now be regarded as "self antigen" $24-26$. A second possibility is that calcium phosphate-antigen conjugates are taken up by mononuclear cells and the calcium phosphate acts as an adjuvant, promoting antigen presentation, while the intestinal mononuclear cell provides tolerogenic signals as these cells are known to be hyporesponsive compared to similar peripheral cells ${ }^{27}$. A third possibility is that the calcium phosphate microparticle directly induces tolerogenic signalling in the antigen-presenting cell through an unknown mechanism. It is now important that this putative pathway of constitutive antigen transfection is established and that the role of luminal calcium phosphate microparticles is delineated.

\section{Exogenous microparticles}

Considerably more is known of exogenous microparticles than it is of endogenous ones. Oral exposure has been reported by Lomer et $a l^{2}$, and amounts to approximately $40 \mathrm{mg} /$ person/ day (or about $10^{12}$ particles) in the UK. Uptake by the Peyer's patch phagocytes has been shown by a number of groups $^{28,29}$ and best characterised by Powell et al ${ }^{3}$. In particular these particles are sub-micron sized and highly resistant to chemical breakdown so, following ingestion and mucosal uptake, they survive both gastrointestinal digestion and cellular processing. They appear to be almost exclusively additives to food, pharmaceuticals and toothpaste and are chiefly aluminosilicates and titanium dioxide, although a small proportion of non-aluminium containing silicates are also found in human gut tissue ${ }^{3,30}$. The distribution of intakes in the UK is shown in Table 1 .

In human gut tissue their dominant occurrence is in basal phagocytes of the Peyer's patch (Fig. 1) where they occur in large numbers.

We hypothesize that exogenous microparticles "hijack" the putative route for endogenous microparticle uptake but, due to their chemically-resistant nature, they cannot then contribute to tolerance induction and may even, in susceptible individuals, establish abnormal immune responsiveness ${ }^{1,31}$. Based upon the capacity of exogenous microparticles for surface adsorption of organic molecules we also anticipate that conjugation of luminal components will occur in the gastrointestinal tract prior to uptake and that this will influence cell responsiveness ${ }^{32}$.

\section{Luminal conjugation}

One important aspect of the gastrointestinal tract is the extent of its secretions and bacterial burden. Secretions are rich in endogenous proteins including immunoglobulins, cations and anions, mucin and other glycoproteins, amino acids and short chain fatty acids. In addition, secretions contain whole and partially digested bacteria from the symbiotic flora and some nonabsorbed food antigens. The bathing of microparticles in such complex fluids surely leads to adsorptive interactions: these particles, which typically have charged, large-area surfaces, could adsorb luminal constituents, facilitated by both ionic and entropic forces. In particular, cations such as calcium can provide bridging charges for larger proteins and glycoproteins to absorb to the surface of particles ${ }^{5-7}$. In model systems we have been able to show that adsorption of a typical

Table 1. *Mean total daily intakes per person in the UK of microparticulate silicates (including aluminosilicates) and titanium dioxide derived from food, pharmaceuticals and toothpaste

\begin{tabular}{lcccc}
\hline & Food (\%) & $\begin{array}{c}\text { Pharmaceuticals } \\
(\%)\end{array}$ & $\begin{array}{c}\text { Toothpaste } \\
(\%)\end{array}$ & $\begin{array}{c}\text { Total intake } \\
(\mathrm{mg} / \text { person/day) }\end{array}$ \\
\hline Silicates & 14.0 & 44.0 & 42.0 & 34.0 \\
$\begin{array}{c}\text { Titanium } \\
\text { dioxide }\end{array}$ & 47.0 & 48.0 & 5.0 & 5.9 \\
\hline
\end{tabular}

${ }^{*}$ Data from Lomer et al. ${ }^{2}$ 


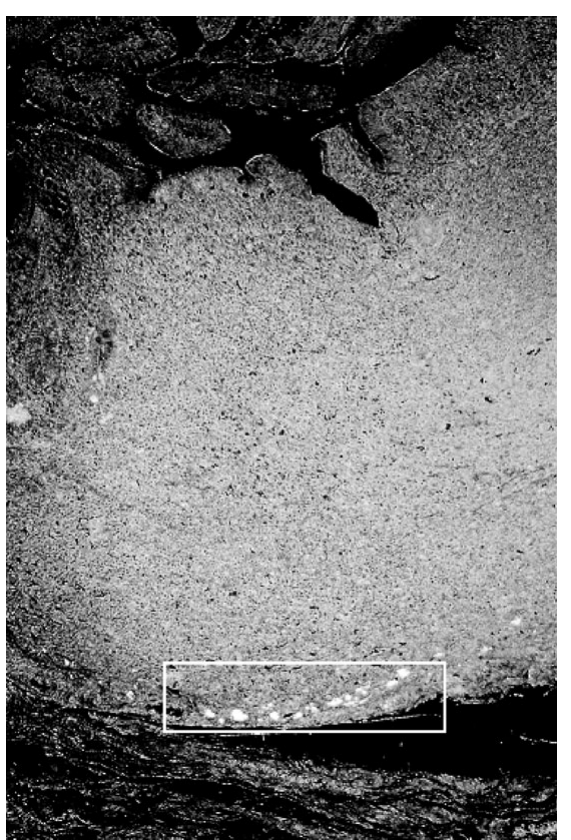

Fig. 1. Dark field microscopy of human small bowel showing an area (in the white box) of particle-containing cells or "pigment cells" (each cell appearing bright white due to reflectance by the pigment in dark field microscopy) located at the base of a human Peyer's Patch (original $\times 200$ ).

luminal constituent (namely bacterial lipopolysaccharide, LPS) by exogenous microparticles (either titanium dioxide or aluminosilicates) occurs in the presence of sufficient calcium ions $^{23,32,33}$. Calcium ions, present in the distal gut lumen, would therefore provide excellent bridging potential for components such as LPS or peptidoglycansaccharide (PGPS), to adsorb to ingested microparticles. It is likely that these LPScalcium-microparticle conjugates are not all pure single particle entities because calcium itself may partially precipitate and complex aggregates may then form ${ }^{32}$. Nonetheless the aggregates still fall well within the microparticle range and are avidly taken up by phagocytic cells ${ }^{32}$. Further work is needed to (a) incubate such particles with real intestinal secretions and gut contents to see which materials are preferentially adsorbed and (b) to look in vivo and confirm this model although, analytically, this is a significant challenge. Nonetheless, it seems most likely that ingested dietary microparticles will adsorb certain luminal materials and, as such, be able to carry them into the intestinal tissue via the M-cell portal in this conjugated fashion. Our subsequent work has investigated what the cellular responses are to native particles or conjugated particles.

\section{Dietary microparticles and cellular activity}

Certain bacterial motifs that are specifically recognised by cells of the immune system are referred to as pathogen associated molecular patterns (PAMPs) and include, for example, LPS (recognised by toll like receptor 4 (TLR 4)), bacterial flagellin (recognised by TLR 5) and PGPS which contains motifs that are recognised by a number of receptors including the intracellular nuclear oligomerisation domains (NODS). NOD2 for example recognises muramyl dipeptide while NOD1 recognises MurNAc-L-Ala-D-Glu-meso-diaminopime- lic acid $^{34}$. Recognition involves specific ligation and cellular responsiveness which typically is an increase in the secretion of pro- inflammatory cytokines such as IL-8, IL-6, TNF- $\alpha$ and IL-1- $\beta$ coupled with potential changes to cell functions such as antigen presentation and maturation/partial differentiation. Recently Blander et al have shown that dendritic cell uptake of antigen-conjugated particles, co-delivered with LPS (i.e. within the same phagolysosomes), led to presentation of the antigen at the cell surface for induction of $\mathrm{T}$ cell proliferative responses ${ }^{26}$. In contrast, in the absence of LPS from this specific compartment, the antigen was not presented. Other PAMPs were not investigated but such work provides a theoretical basis for the immune system to differentiate between "inert particles" and biologically active particles such as invasive bacteria. Moreover, it provides a basis for distinction between particulate self-antigen such as apoptotic cells and particulate bacterial antigen such as in early infection. Thus the immune system can determine, based on recognition of these PAMPs, whether to mount a response through $\mathrm{T}$ cell activation or not. We thus recognise the importance in understanding how particles and PAMPs engage with the acquired immune system in our work but have so far restricted our studies to outcomes of the innate immune system (i.e. cytokines). The following is a brief review of these works.

In initial work we used intestinal organ cultures (human gut biopsies in culture) as a model for assessment of dietary microparticle effects on stimulation of the pro-inflammatory cytokine IL1- $\beta^{33}$. Microparticles alone (titanium dioxide) had no effect, neither did high dose LPS. It is important to recognize that immune cells of the gut are hypo-responsive to LPS as indeed they are to PAMPs in general from what is understood ${ }^{27}$. So unlike with peripheral cells, for example, this lack of gut cell responsiveness to LPS is expected. In contrast when LPS and dietary microparticles were added together in the presence of excess calcium, a small (1.5 to 4 fold) increase in IL1- $\beta$ secretion was observed. The same pattern was observed using peripheral blood mononuclear cells except this time the increase was dramatic with the LPS-calcium-titanium dioxide conjugate (30 to 60 fold above base line $)^{32}$. While this again emphasizes the tolerant nature of intestinal cells it also demonstrates that such particles may have immune potentiating possibilities when bound with PAMPs from the gastrointestinal lumen. Further work really needs to dissect out the effects of calcium in the system because we have subsequently shown that this is an important, even major, part of the activity of the conjugate/agglomerate ${ }^{23}$. Nonetheless this is still likely to represent in vivo situations.

There are a number of problems with organ cultures of colonic biopsies including viability and surface area for full exposure in particle-based studies. Thus, subsequently, we continued this work using isolated human intestinal cells from the lamina propria. We also undertook time course studies. The results were very similar in that there was a small but significant immune potentiating effect of the conjugates versus the individual agents, namely titanium dioxide and lipopolysaccharide alone ${ }^{23}$. However, the importance of the calcium was especially emphasized because this alone was able to induce some significant secretion of IL1- $\beta$, albeit not as protracted as for the conjugate. Overall, the effects were associated with the induction of cell death in the phagocytic population suggesting that caspase activity 
had been increased ${ }^{23,32}$. Caspase 1 , for example, is required for the cleavage of Pro IL1- $\beta$ and using inhibition-based studies we have been able to show that activation of this caspase was required for the IL1- $\beta$ release. Moreover, unlike in the previous work with organ cultures, we were able to show significant differences in the response of the cells from patients with inflammatory bowel disease (IBD) versus controls. This was clearly related to the baseline inflammatory status of the patients and probably was not therefore a specific hyper-responsiveness in IBD. It is unclear why this was not observed in the organ culture work but may represent the different genotypes (which were not tested in either works) that were present in the relatively small populations tested or due to the different techniques used.

In further studies we were able to confirm that the results described above were a specific effect of particulate uptake, which was mediated via the scavenger receptor, and was associated with increases in secretion of a large number of pro-inflammatory cytokines albeit only with IL-1 $\beta$ did we see a synergistic effect between particle plus calcium plus LPS compared to the additive effect of the individual components $^{23,32}$. These data suggested to us that such ingested microparticle-conjugates/agglomerates may exacerbate inflammation in the permeable and hyper responsive gut of subjects with inflammatory bowel disease.

In an initial small pilot study we were able to show that withdrawal of microparticles from the diet of patients with Crohn's disease was associated with marked improvement of symptoms ${ }^{35}$. This stimulated a large multi-centre double blind trial, which was carefully undertaken. In this work however we were able to show no effect of microparticle withdrawal on disease activity or objective biochemical measures of disease $^{36}$. Patients had to be concomitantly treated with immunosuppressant drugs (corticosteroids in this case) so the differences observed were between corticosteroids plus the normal microparticle-containing diet versus corticosteroids plus a low microparticle diet. It is possible that the corticosteroids masked any differences but, in which case, the differences must have been subtle. A second problem was that despite careful choice of drugs and dietary advice, patients were still being exposed to some microparticles and it may be that a microparticle-free diet, rather than a low microparticle diet, is what is required. However, the more likely interpretation is that microparticles do not significantly influence the pathogenesis of Crohn's disease. Whether they are involved as aetiological factors in triggering the disease is a different matter and something we continue to investigate.

Our further work will look at the effects of microparticles on the acquired immune system and in patients of different genotypic status- for example, in those carrying NOD2 variants. We cannot get away from the fact that Crohn's disease is a modern, Western disease, the first signs of which are seen overlying intestinal lymphoid aggregates and that there is clearly a gene-environment interaction in the triggering of the disorder. The presence of these microparticles in the modern, Western diet, and their uptake and large quantitative presence within the intestinal lymphoid aggregates of the gut still makes them tantalizing candidates for involvement in this complex polygenic disorder. Evidence however remains elusive.

\section{Conflict of interest statement}

The authors have no conflict of interest to report. The authors contributed equally in the preparation and redaction of the manuscript.

\section{References}

1. Lomer MC, Thompson RP \& Powell JJ (2002) Fine and ultrafine particles of the diet: influence on the mucosal immune response and association with Crohn's disease. Proc Nutr Soc 61, $123-130$.

2. Lomer MC, Hutchinson C, Volkert S, Greenfield SM, Catterall A, Thompson RP \& Powell JJ (2004) Dietary sources of inorganic microparticles and their intake in healthy subjects and patients with Crohn's disease. Br J Nutr 92, 947-955.

3. Powell JJ, Ainley CC, Harvey RS, Mason IM, Kendall MD, Sankey EA, Dhillon AP \& Thompson RP (1996) Characterisation of inorganic microparticles in pigment cells of human gut associated lymphoid tissue. Gut 38, 390-395.

4. Ellingsen JE (1991) A study on the mechanism of protein adsorption to TiO2. Biomaterials 12, 593-596.

5. Govers MJ, Termont DS, Van Aken GA \& Van der Meer R (1994) Characterization of the adsorption of conjugated and unconjugated bile acids to insoluble, amorphous calcium phosphate. J Lipid Res 35, 741-748.

6. Lapre JA, De Vries HT, Koeman JH \& Van der Meer R (1993) The antiproliferative effect of dietary calcium on colonic epithelium is mediated by luminal surfactants and dependent on the type of dietary fat. Cancer Res 53, 784-789.

7. Van der Meer R, Welberg JW, Kuipers F, Kleibeuker JH, Mulder NH, Termont DS, Vonk RJ, De Vries HT \& De Vries EG (1990) Effects of supplemental dietary calcium on the intestinal association of calcium, phosphate, and bile acids. Gastroenterology 99, 1653-1659.

8. Powell JJ, Whitehead MW, Ainley CC, Kendall MD, Nicholson JK \& Thompson RP (1999) Dietary minerals in the gastrointestinal tract: hydroxypolymerisation of aluminium is regulated by luminal mucins. J Inorg Biochem 75, 167-180.

9. Schedl HP, Osbaldiston GW \& Mills IH (1968) Absorption, secretion, and precipitation of calcium in the small intestine of the dog. Am J Physiol 214, 814-819.

10. Favus MJ (1985) Factors that influence absorption and secretion of calcium in the small intestine and colon. Am J Physiol 248, G147-G157.

11. Sass W, Dreyer HP \& Seifert J (1990) Rapid insorption of small particles in the gut. Am J Gastroenterol 85, 255-260.

12. Sernka TJ \& Borle AB (1969) Calcium in the intestinal contents of rats on different calcium diets. Proc Soc Exp Biol Med 131, $1420-1423$.

13. Pappo J \& Ermak TH (1989) Uptake and translocation of fluorescent latex particles by rabbit Peyer's patch follicle epithelium: a quantitative model for M cell uptake. Clin Exp Immunol 76, 144-148.

14. Smith MW, Thomas NW, Jenkins PG, Miller NG, Cremaschi D \& Porta C (1995) Selective transport of microparticles across Peyer's patch follicle-associated $\mathrm{M}$ cells from mice and rats. Exp Physiol 80, 735-743.

15. Pele L \& Powell JJ Microparticles: A link between Modern Life and Inflammatory Bowel Disease (2005) In Inflammatory Bowel Disease: Translation from Basic Research to Clinical Practice, 140 ed., pp. 123-137 [JF Colombel, C Gasche, J Scholmerich and B Vucelic, editors]. Dordrecht, The Netherlands: Springer.

16. Lupton JR, Steinbach G, Chang WC, et al. (1996) Calcium supplementation modifies the relative amounts of bile acids 
in bile and affects key aspects of human colon physiology. J Nutr 126, 1421-1428.

17. Bovee-Oudenhoven IM, Lettink-Wissink ML, Van Doesburg W, Witteman BJ \& Van Der Meer R (2003) Diarrhea caused by enterotoxigenic Escherichia coli infection of humans is inhibited by dietary calcium. Gastroenterology 125, 469-476.

18. Bovee-Oudenhoven IM, Termont DS, Weerkamp AH, FaassenPeters MA \& Van der Meer R (1997) Dietary calcium inhibits the intestinal colonization and translocation of Salmonella in rats. Gastroenterology 113, 550-557.

19. Bovee-Oudenhoven IM, Wissink ML, Wouters JT \& Van der Meer R (1999) Dietary calcium phosphate stimulates intestinal lactobacilli and decreases the severity of a salmonella infection in rats. J Nutr 129, 607-612.

20. Hambly RJ, Saunders M, Rijken PJ \& Rowland IR (2002) Influence of dietary components associated with high or low risk of colon cancer on apoptosis in the rat colon. Food Chem Toxicol 40, 801-808.

21. Penman ID, Liang QL, Bode J, Eastwood MA \& Arends MJ (2000) Dietary calcium supplementation increases apoptosis in the distal murine colonic epithelium. J Clin Pathol 53, 302-307.

22. Zhu Y, Mahon BD, Froicu M \& Cantorna MT (2005) Calcium and 1 alpha,25-dihydroxyvitamin D3 target the TNF-alpha pathway to suppress experimental inflammatory bowel disease. Eur J Immunol 35, 217-224.

23. Evans SM, Ashwood P, Warley A, Berisha F, Thompson RP \& Powell JJ (2002) The role of dietary microparticles and calcium in apoptosis and interleukin-1beta release of intestinal macrophages. Gastroenterology 123, 1543-1553.

24. Ferguson TA, Herndon J, Elzey B, Griffith TS, Schoenberger S \& Green DR (2002) Uptake of apoptotic antigen-coupled cells by lymphoid dendritic cells and cross-priming of CD8(+) T cells produce active immune unresponsiveness. J Immunol 168, 5589-5595.

25. Liu K, Iyoda T, Saternus M, Kimura Y, Inaba K \& Steinman RM (2002) Immune tolerance after delivery of dying cells to dendritic cells in situ. J Exp Med 196, 1091-1097.
26. Blander JM \& Medzhitov R (2006) Toll-dependent selection of microbial antigens for presentation by dendritic cells. Nature 440, 808-812.

27. Smythies LE, Sellers M, Clements RH, Mosteller-Barnum M, Meng G, Benjamin WH, Orenstein JM \& Smith PD (2005) Human intestinal macrophages display profound inflammatory anergy despite avid phagocytic and bacteriocidal activity. $J$ Clin Invest 115, 66-75.

28. Shepherd NA, Crocker PR, Smith AP \& Levison DA (1987) Exogenous pigment in Peyer's patches. Hum Pathol 18, 50-54.

29. Urbanski SJ, Arsenault AL, Green FH \& Haber G (1989) Pigment resembling atmospheric dust in Peyer's patches. Mod Pathol 2, 222-226.

30. Mazumder RN, Bode J, McIntyre MM \& Ghosh S (2002) Detection of chromium microparticles in inflammatory bowel disease (IBD) tissues by energy dispersive analysis of X-rays. Gut 50, 83A.

31. Powell JJ, Harvey RS \& Thompson RP (1996) Microparticles in Crohn's disease-has the dust settled? Gut 39, 340-341.

32. Ashwood P, Thompson RP \& Powell JJ (2007) Fine particles that adsorb lipopolysaccharide via bridging calcium cations may mimic bacterial pathogenicity towards cells. Exp Biol Med 232, 107-117.

33. Powell JJ, Harvey RS, Ashwood P, Wolstencroft R, Gershwin ME \& Thompson RP (2000) Immune potentiation of ultrafine dietary particles in normal subjects and patients with inflammatory bowel disease. J Autoimmun 14, 99-105.

34. Netea MG, Ferwerda G, de Jong DJ, et al. (2005) The frameshift mutation in Nod2 results in unresponsiveness not only to Nod2- but also Nod1-activating peptidoglycan agonists. $J$ Biol Chem 280, 35859-35867.

35. Lomer MC, Harvey RS, Evans SM, Thompson RP \& Powell JJ (2001) Efficacy and tolerability of a low microparticle diet in a double blind, randomized, pilot study in Crohn's disease. Eur J Gastroenterol Hepatol 13, 101-106.

36. Lomer MC, Grainger SL, Ede R, et al. (2005) Lack of efficacy of a reduced microparticle diet in a multi-centred trial of patients with active Crohn's disease. Eur J Gastroenterol Hepatol 17, 377-384. 